\title{
PET/MRI for Neurologic Applications
}

\author{
Ciprian Catana ${ }^{1}$, Alexander Drzezga ${ }^{2}$, Wolf-Dieter Heiss ${ }^{3}$, and Bruce R. Rosen ${ }^{1}$ \\ ${ }^{I}$ Athinoula A. Martinos Center for Biomedical Imaging, Department of Radiology, Massachusetts General Hospital and Harvard \\ Medical School, Charlestown, Massachusetts; ${ }^{2}$ Nuklearmedizinische Klinik und Poliklinik der Technischen Universität München, \\ Klinikum rechts der Isar, Munich, Germany; and ${ }^{3}$ Max Planck Institute for Neurological Research, Cologne, Germany
}

\begin{abstract}
PET and MRI provide complementary information in the study of the human brain. Simultaneous PET/MRI data acquisition allows the spatial and temporal correlation of the measured signals, creating opportunities impossible to realize using stand-alone instruments. This paper reviews the methodologic improvements and potential neurologic and psychiatric applications of this novel technology. We first present methods for improving the performance and information content of each modality by using the information provided by the other technique. On the PET side, we discuss methods that use the simultaneously acquired MRI data to improve the PET data quantification. On the MRI side, we present how improved PET quantification can be used to validate several MRI techniques. Finally, we describe promising research, translational, and clinical applications that can benefit from these advanced tools.

Key Words: simultaneous PET/MRI; multimodal imaging; neurology
\end{abstract}

J Nucl Med 2012; 53:1916-1925

DOI: 10.2967/jnumed.112.105346

\section{$\mathbf{M}$}

RI and PET provide complementary anatomic, physiologic, metabolic, and functional information about the brain. Pooling information obtained with each modality has long been performed through a parallel analysis of the sequentially acquired data and, more commonly today, by using software coregistration techniques. However, underlying such studies is the assumption that no significant changes in physiologic or cognitive conditions have occurred between the 2 examinations. Although a good assumption for some studies, this may not be the case more generally. For example, a subject's mental state may change on time frames from minutes to even seconds while physiologic and metabolic changes can occur on the order of minutes in some disease conditions such as acute ischemic stroke or migraine. Likewise, rapid changes in baseline phys-

Received Aug. 15, 2012; revision accepted Oct. 19, 2012.

For correspondence or reprints contact: Ciprian Catana, Athinoula A. Martinos Center for Biomedical Imaging, Department of Radiology, Massachusetts General Hospital and Harvard Medical School, Building

149, Room 2301, 13th St., Charlestown, MA 02129.

E-mail: ccatana@nmr.mgh.harvard.edu

Published online Nov. 9, 2012.

COPYRIGHT (C) 2012 by the Society of Nuclear Medicine and Molecular Imaging, Inc. iology can occur with some therapeutic interventions, such as neurooncologically directed treatments with antiangiogenic agents.

One means to address such potential pitfalls is through the simultaneous collection of MRI and PET data. The feasibility of simultaneous PET and MRI data acquisition for human studies was first demonstrated in 2007, and proof-of-principle brain data (Fig. 1) were collected using a prototype MRI-compatible PET insert-called BrainPETpositioned inside a commercially available 3-T MRI Trio system (Siemens Healthcare Inc.) (1). In 2010, a fully integrated PET/MRI scanner also became available for human whole-body imaging (Biograph mMR; Siemens) (2).

Simultaneous PET/MRI allows for both spatial and temporal correlation of the signals, creating opportunities impossible to realize using sequentially acquired data. The features of this new technology may be particularly appealing to applications in neuroscience and translational neurologic and psychiatric research, considering that MRI represents the first-line diagnostic imaging modality for numerous indications and that a great number of specific PET tracers are available today to assess functional and molecular processes in the brain. In the study of physiologic brain function, simultaneous acquisition may allow improved in vivo assessment and cross-correlation of several neuropsychologic events, such as changes in hemodynamics, including quantitative assessment of cerebral blood flow (CBF), volume (CBV), and oxygenation; neurovascular coupling; inflammation and microglial activation; ischemia; necrosis; and apoptosis. The understanding of the pathophysiologic mechanisms underlying disease states and the clinical evaluation of various disorders of the central nervous system over time might also be improved, such as quantitative measurements of pathologic processes in brain cancer before and after treatment; neurodegenerative diseases; and acute and chronic stroke, including neuroplasticity associated with stroke recovery. In addition, other disorders associated with changes in mental status, such as depression, dementia, schizophrenia, and obsessivecompulsive disorders, might be investigated in new ways, combining anatomic, functional, and metabolic measurements during identical examination conditions.

In this article, we discuss the methodologic opportunities created by simultaneous PET/MRI data acquisition and 


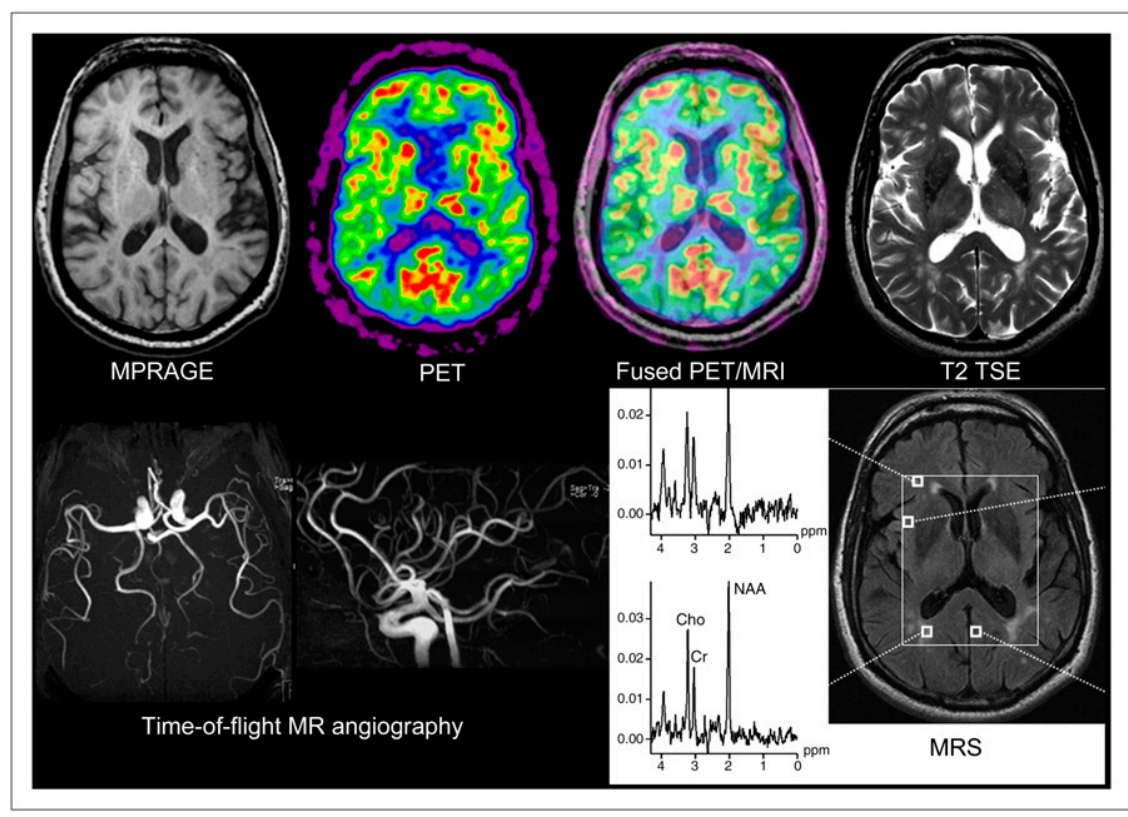

FIGURE 1. First simultaneous PET/MRI study in 66-y-old healthy volunteer. MRI sequences included T2-weighted turbo spin echo, echo planar, time-of-flight MR angiography, and MR spectroscopy. PET image displayed was reconstructed from 20-min emission data recorded at steady state after injection of $370 \mathrm{MBq}$ of ${ }^{18} \mathrm{~F}-\mathrm{FDG}$. Data were acquired on BrainPET prototype (Siemens). MPRAGE $=$ magnetization-prepared rapid gradient echo; MRS = MR scintigraphy; TSE $=$ turbo spin echo. (Reprinted with permission of (1).) describe how this novel imaging modality might benefit neurologic and psychiatric applications.

\section{METHODOLOGIC AND SCIENTIFIC ADVANTAGES}

Simultaneous acquisition immediately brings to mind the possibility of improving the performance and information content of one instrument using the information obtained from the other instrument. This street does not need to be 1-way: the perspective to combine the strengths of both techniques for mutual improvement appears tempting. The accuracy of the PET estimates might be improved by including the MRI information as the structural framework underlying the distribution of the PET signal. Reciprocally, the strength of PET to provide absolute quantitative information might help validate several MRI techniques in vivo. Next, we give examples of methodologic improvements made possible via simultaneous acquisition.

\section{MRI-Based PET Motion Correction}

Subject head motion is difficult to avoid, leading to PET image blurring that could even offset the benefit of using high-resolution scanners. Various techniques have been proposed for restraining the subject's head (with limited success) or for correcting for head movements (3-11). However, most of these methods require a relatively unobstructed view of the optical sensors from outside the scanner-not typically the case on an integrated PET/MRI scanner because of the radiofrequency coils.

In an integrated scanner, the MRI data acquired simultaneously with the PET data can be used to obtain hightemporal-resolution rigid-body motion estimates, most often in the background of the actual MRI data acquisition. One such method consists of repeatedly acquiring anatomic data and coregistering the individual MRI volumes. When fast imaging with EPI or spiral sequences is performed, such methods provide temporal updating of spatial informa- tion in times typically from 2 to $6 \mathrm{~s}$. These methods are particularly attractive because they allow the simultaneous acquisition of functional MRI (fMRI) and PET data, which is of interest for several research applications. For more conventional acquisition methods (e.g., high-resolution anatomic imaging), motion estimates with very high temporal resolution (e.g., every $20 \mathrm{~ms}$ ) can be obtained using embedded navigator pulses (12). Their potential use to correct the PET data in very short frames could be particularly important for performing motion correction in the early phases of a dynamic PET study, when frames as short as $1 \mathrm{~s}$ are often used to sample the radiotracer arterial input function (AIF).

Proof-of-principle MRI-assisted PET rigid-body motion correction studies have already been performed on healthy volunteers using the BrainPET scanner (Fig. 2) (13). MRIbased motion correction has the potential to improve PET as a quantitative method. First, the nominal spatial resolution of the current state-of-the-art scanners can be achieved. Second, the mismatch between the attenuation and emission volumes can be eliminated, assuming that the former was also derived from the simultaneously acquired MRI data (14). Third, better estimates of the radiotracer AIF might be obtained using image-based approaches from the motion-corrected data. Together, these methods might increase the reliability and reproducibility of the PET data, thus potentially benefiting several neurologic applications that require precise quantification (e.g., neuroreceptor studies) or that involve uncooperative subjects (e.g., Alzheimer disease, movement disorders, or pediatric patients).

\section{Partial-Volume Effect (PVE) Correction}

PVEs lead in PET to an under- or overestimation of tissue activity concentrations that depends on the activity distribution and the size and shape of the structures of interest (15). As a general rule, the signal is not accurately 


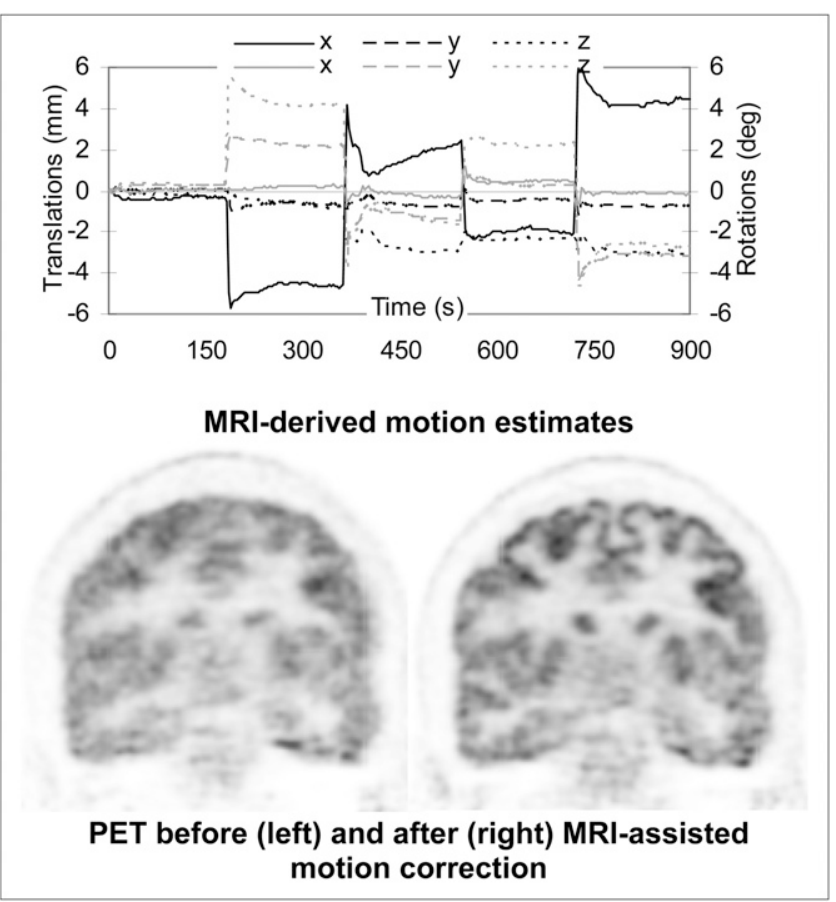

FIGURE 2. MRI-assisted PET motion correction in healthy volunteer using echo planar imaging-derived motion estimates. (Top) Plots of motion estimates: translations along (black) and rotations about (gray) 3 orthogonal axes. (Bottom) PET data reconstructed before (left) and after (right) motion correction. Substantial improvement in image quality can be observed after correction. Data were acquired on BrainPET prototype at Martinos Center, Massachusetts General Hospital.

recovered in structures smaller than 2-3 times the fullwidth-at-half-maximum spatial resolution of the scanner. Because the spatial resolution of the current state-of-theart clinical PET scanners is comparable to the cortical gray matter thickness (i.e., $2-3 \mathrm{~mm}$ ), the activity concentration in these structures is clearly affected by PVEs in virtually all neurologic studies performed today (Fig. 3).

Several algorithms have been proposed for correcting for PVEs. Some methods apply the correction to the measured values derived from regions of interest (16-19) or attempt to apply the correction on a voxel-by-voxel basis (20). Other methods use the anatomic information provided by MRI as prior information to regularize the PET images in statistical reconstruction algorithms $(21-23)$ and thus to model the PVEs in the reconstruction. Although these methods have been used for numerous clinical studies, none has been widely accepted because many factors affect the accuracy of PVE correction: segmentation of structures of interest from the anatomic MRI data, spatial coregistration of the MRI and PET volumes, characterization of the scanner's point-spread function, and the assumptions made during the correction (23-25). The spatial coregistration problems are eliminated in the case of an integrated PET/MRI system, for which simultaneous acquisition guarantees spatial correlation. Furthermore, head motion, which is an additional

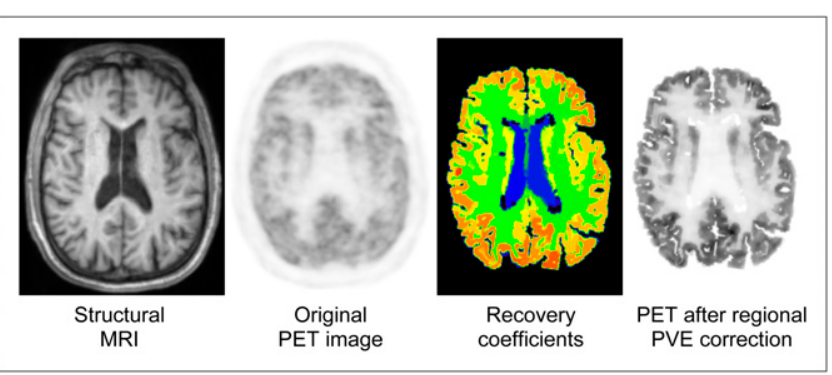

FIGURE 3. Influence of MRI-based PVE correction on PET image contrast for normal brain. From left to right are shown MRI image used to automatically segment ROls of multiple brain structures, original PET image, PVE correction factors for mean ROI values calculated via geometric transfer matrix method using segmented $\mathrm{MRI}$ and original PET images as inputs, and original PET image after application of recovery coefficients. Data were acquired on BrainPET prototype at Martinos Center, Massachusetts General Hospital. (Courtesy of Spencer Bowen.)

source of image blurring, can be minimized using MRI-based motion correction.

\section{Image-Based Radiotracer AIF Estimation}

Accurate PET quantification requires an input function (i.e., plasma time-activity curve of tracer delivery to the tissue) to the compartment models used for estimating parameters of interest related to normal and pathologic changes in tissue function or metabolism. The gold standard for determining the AIF involves radial artery catheterization, which limits usefulness in routine clinical PET studies. Alternatively, less invasive methods such as arterialized venous blood sampling, or noninvasive techniques such as those that are image-based or population-based, have been proposed (26).

One image-based approach is to derive the AIF from a region of interest placed across blood vessels after tracer administration. Correct definition of the region of interest over the vessel and correction for confounding effects such as spill-in from adjacent tissues or spill-out in the case of relatively small vessels can be challenging using only the PET images. In a combined scanner, the anatomic and physiologic MRI information might be used for this purpose. The coregistered MRI anatomic images can be used to accurately determine the position and the size of the vessels of interest (e.g., from the MR angiography data). With coadministration of both MRI contrast and PET tracers, MRI might also provide additional information about the dynamics of bolus delivery to the tissue of interest and assess any local changes in blood flow, potentially reducing the problems of bolus delay and dispersion inherent in the global AIF estimate. For some radiotracers, however, the AIF may still not be a suitable predictor of the input function of the parent radiolabeled compound, and additional information about metabolites may be required.

\section{Technique Cross-Calibration and Validation}

Cerebral Perfusion. $\mathrm{H}_{2}{ }^{15} \mathrm{O}$ PET is considered the gold standard for measuring CBF, but a variety of MRI methods 
have also emerged for the same purpose, including dynamic susceptibility contrast imaging (27) and arterial spin labeling (28). Perhaps not surprisingly, there are notable discrepancies between PET and MRI CBF measures (29) (as well as between the individual MRI methods), but the degree to which these discrepancies represent a true technique variation, versus differences in physiologic state between distinct measurement periods, is unknown. Thus, the use of simultaneous PET/MRI for an initial cross-validation of different techniques offers a unique means to begin to address confounds associated with this fundamental physiologic measurement. Furthermore, beyond perfusion, both PET and MRI can be used independently to assess $\mathrm{CBV}$, cerebral metabolic rate of oxygen, and oxygen extraction fraction. Given the overlapping abilities to perform these measurements with MRI-based methods, a combined instrument might simultaneously perform multiple comparisons, cross-validating several physiologic measurements.

Neuronal Activation. During periods of increased (or decreased) neural activation, a variety of physiologic states are known to change, including both hemodynamic and metabolic parameters. These changes, particularly in CBF and $\mathrm{CBV}$, have been assessed for many years by PET to characterize regional brain activity, and similar functional studies are now possible with MRI $(30,31)$. One interesting observation from early PET experience is that the increase in $\mathrm{CBF}$ was shown to be greater than the increase in the cerebral metabolic rate of oxygen (32). The decrease in the concentration of paramagnetic deoxyhemoglobin in the venous blood as a result of the increase in venous blood oxygenation level is the basis of the increased blood oxygen level-dependent (BOLD) signal intensity (33), which is most typically used for human fMRI studies. The decrease in the cerebral oxygen extraction fraction can also be measured using ${ }^{15} \mathrm{O}_{2}$ PET. Combined PET/MRI allows for the validation and detailed quantitative modeling of the relationship between oxygen extraction fraction, $\mathrm{CBV}, \mathrm{CBF}$, and BOLD signals and perhaps might reveal new aspects not obvious when the experiments are performed using separate instruments at different times. A simultaneous measurement of $\mathrm{H}_{2}{ }^{15} \mathrm{O}$ PET activation and fMRI would also permit a more selective differentiation of local responses in the tissue (CBF PET) from those in the vascular system (BOLD or CBF MRI). Such data should permit a better understanding of the coupling between regional neuronal activity, changes in $\mathrm{CBF}$ and $\mathrm{CBV}$, and oxygen metabolism.

Brain Baseline State. The concept of baseline state is an essential, though often overlooked, aspect of brain activation studies because the change in signal reported in response to a stimulus (measured either with PET or MRI techniques) is typically assessed with respect to an (unquantified) baseline condition. Furthermore, identifying a physiologic baseline state is extremely relevant for assessing pathologic changes in several conditions (e.g.,
Alzheimer disease, head trauma, stroke, and neuropsychiatric disorders). PET and MRI techniques have been proposed for studying many aspects of this complex state. For example, the CBF measurements mentioned above (e.g., $\mathrm{H}_{2}{ }^{15} \mathrm{O}-\mathrm{PET}$ and arterial spin labeling MRI) can be used to obtain precise measurement of the resting cerebral perfusion, measurements of the cerebral metabolic rate of glucose and cerebral metabolic rate of oxygen can inform us about the basal metabolism, and oxygen extraction fraction can be used to provide a functional baseline $(34,35)$. Even with these advanced methods, brain activity at rest is far from being completely understood. An integrated PET/MRI scanner offers the unique opportunity to simultaneously quantify and correlate the physiologic, metabolic, and functional baselines of the brain.

Brain Connectivity Measures. The brain remains the organ about which we know the least regarding the fundamental relationship between its structure and its function, in large part because methods to map the complex organizational relationships between its component parts in vivo have not existed. This is beginning to change. For example, diffusion MRI allows the identification of white matter orientation locally, permitting estimates of white matter connectivity between brain regions. In addition to information on structural connectivity, resting-state fMRI procedures allow the identification of functional connectivity between different brain areas through the observation of the interregional correlation of physiologic noise on time scales of seconds (36). What is missing in these measurements is of course the role of specific neurotransmitters. Adding information on transmitter release and receptor occupancy, as well as on metabolism in connected areas of functional networks, may be of special interest.

Such data are of more than just academic interest. It may be of great clinical relevance to study the state of functional connectivity together with functional PET markers. Recent studies were already able to demonstrate a regional interrelation between hypometabolism $\left({ }^{18} \mathrm{~F}-\mathrm{FDG}\right.$ PET) and disruption of functional connectivity in patients at risk for Alzheimer disease (37). As another example, diffusion tractography, which can provide important clinical information on brain tumors by revealing the displacement and integrity of peritumoral fiber tracts, may — combined with PET ligand data on the location of vital tumor tissue-be highly important for treatment planning. Identification of epileptic foci can be confirmed with confidence through the confluence of connectional and metabolic data. Ultimately, the diagnosis of developmental "connectopathies" such as autism and schizophrenia, and other behavioral disorders, may come within reach of this approach.

\section{ADVANTAGES OF SIMULTANEOUS PET/MRI IN CLINICAL AND RESEARCH APPLICATIONS}

Simultaneous PET/MRI is expected to be a more quantitatively accurate tool than either method alone, and several diseases may yield their secrets best to highly quantitative 
methods. This advantage alone could have a positive impact on all clinical and research applications that require improved quantification.

One practical advantage that cannot be overstated is that because most patients undergoing PET for neuropsychiatric diseases typically also undergo MRI as part of their routine care, the option to perform the 2 examinations at the same time in the same scanner represents a significant increase in patient comfort. This 1-stop shop decreases the number of visits the patient must make to the imaging departments and reduces the overall imaging time.

Although technical challenges still exist, particularly on the PET side (e.g., MRI-based attenuation correction is still an area of active research), the goal of the scanner manufacturers is to combine the 2 devices without compromising their individual performance. Indeed, all the standard and advanced MRI methods for assessing brain anatomy-physiologic parameters, water diffusion, metabolite concentrations, and the like-are available in a combined scanner and can be profitably combined with PET methods (Fig. 1) using proven and novel radiotracers.

Next, without aiming to comprehensively review the usefulness of all MRI and PET techniques for all potential applications, we describe applications that might take advantage of the improved quantification or cross-calibrated measurements and we provide examples of how one might use simultaneous PET/MRI to further our knowledge of these fields. In each case, we first describe some of the limitations of the existing techniques. We then discuss how simultaneous PET/MRI might help from a methodologic point of view, and we cautiously speculate on the potential clinical impact.

\section{Brain Tumors}

MRI is firmly established as a diagnostic and assessment method of choice for brain tumor patients and has found increasing use as a cancer imaging biomarker (38-41). Several quantitative MRI methods (e.g., dynamic contrast-enhanced MRI, dynamic susceptibility contrast MRI, MR spectroscopy, and diffusion MRI) have been used to improve cancer imaging. However, these MRI techniques also have limitations. Dynamic contrast-enhanced MRI, for example, provides a marker of enhancement, but enhancement depends on $\mathrm{CBF}$ and $\mathrm{CBV}$, the permeability of the capillary bed, and the surface area of the capillary bed (42), and sorting out which of these is changing after a given treatment can be challenging (43). Furthermore, contrast enhancement in the brain is dependent on the integrity of the blood-brain barrier. PET tracers for studying amino acid transport (e.g., ${ }^{11} \mathrm{C}$-methionine and ${ }^{18} \mathrm{~F}$-fluoroethyltyrosine), cellular proliferation (e.g., ${ }^{18}$ F-fluorothymidine), and tissue hypoxia (e.g., ${ }^{18}$ F-fluoromisonidazole) (Fig. 4) have been demonstrated to have the potential to circumvent several of the existing limitations of MRI for brain tumor diagnosis. However, the PET estimates are also influenced by permeability or other delivery-related factors that might be better under- stood using MRI techniques. For example, separating the ${ }^{18}$ F-fluorothymidine transport and metabolic trapping components $(44,45)$ and comparing these with the permeability changes assessed with MRI might provide insights beyond those provided by either modality alone. As another example, the importance of assessing tumor perfusion and hypoxia independently was demonstrated using a dynamic model for analyzing dynamic ${ }^{18} \mathrm{~F}$-fluoromisonidazole PET data (46), and MRI offers the ability to directly quantify brain and tumor perfusion for inclusion in PET kinetic modeling. All these studies would greatly benefit from the improved PET data quantification obtained using the simultaneously acquired MRI information.

Glioblastoma, the most aggressive and uniformly fatal brain tumor, is a disease in desperate need of successful therapies, and quantitative imaging biomarkers are required for evaluating the effects and understanding the mechanisms of action of novel therapeutic agents (e.g., antiangio-

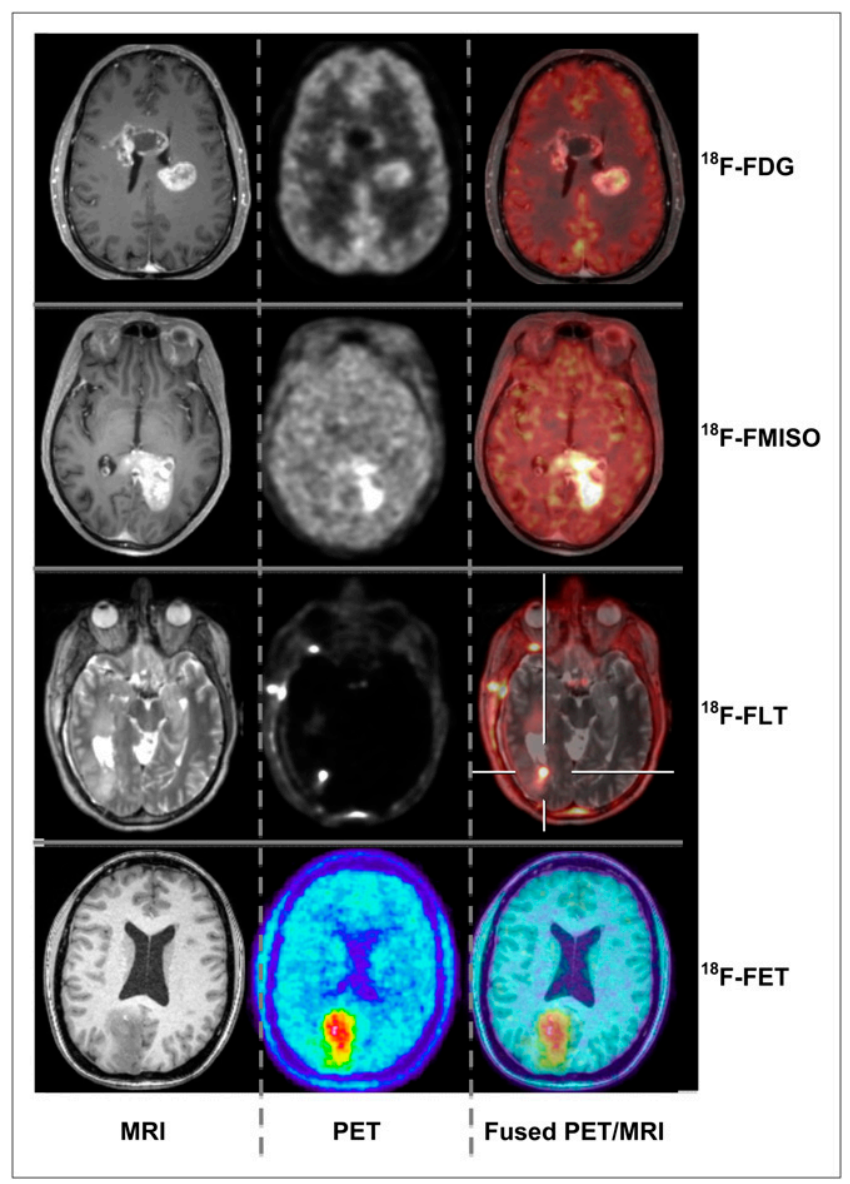

FIGURE 4. Simultaneous PET/MRI studies in patients with brain tumors. From left to right are shown axial MRI, PET, and fused images for different tracers: ${ }^{18} \mathrm{~F}-\mathrm{FDG},{ }^{18} \mathrm{~F}$-fluoromisonidazole (FMISO), ${ }^{18} \mathrm{~F}$-fluorothymidine (FLT) (data were acquired on BrainPET prototype at Martinos Center, Massachusetts General Hospital) and ${ }^{18} \mathrm{~F}$-fluoroethyltyrosine (FET) (data were acquired on BrainPET prototype at Forschungszentrum Juelich). (Images courtesy of Hans Herzog and Karl-Josef Langen.) 
genic agents $(47,48))$. Simultaneous MRI measurements of microvascular proliferation, permeability, and PET tracer uptake might help quantify precisely how tumor proliferation, tumor vascular properties, and antitumor effects occur and interact, thus enabling better understanding of tumor biology, evolution, and therapeutic response on an individual basis.

In combination, PET and MRI data should allow improved diagnostic accuracy and might be used for surgery and radiation therapy planning (49). It has been demonstrated that the combination of both modalities can change the target volume used for radiation therapy, potentially having a significant effect on patient outcome (50). An integrated imaging device becomes especially important in the planning of radiation therapy or surgical intervention when brain areas of critical function in the vicinity of tumors must be identified (51).

\section{Dementia/Neurodegeneration}

In the work-up of patients with cognitive impairment or suspected dementia, morphologic procedures (i.e., CT or MRI) are used to exclude nonneurodegenerative and potentially treatable causes for the symptoms. Recently, novel MRI techniques have emerged that go beyond this use. These include quantitative MRI morphology (52-56), diffusion tensor imaging $(57,58)$, fMRI $(59,60)$, and methods that allow the assessment of changes in the resting-state functional connectivity networks of the brain, such as the default mode network $(61,62)$. However, these tools have not yet been proven to provide high diagnostic sensitivity and specificity. PET findings, on the other hand, allow more reliable diagnosis and differential diagnosis of dementing disorders, as well as prediction in predementia stages. These include specific patterns of regional glucose metabolism indicating neuronal dysfunction (Fig. 5) (63) and changes in neurotransmitter systems (e.g., cholinergic, serotonergic, or dopaminergic) (63-66). Recently, new tracers for molecular imaging of $\beta$-amyloid plaques $(67,68)$ have been introduced. Many modern therapy approaches are directed toward the prevention or removal of this pathology, and thus, expectations about these PET tracers with regard to early diagnosis, patient selection, and therapy monitoring are high.

However, PET estimates in small and anatomically complex structures (such as those first affected in the progression of neurodegenerative disorders) are biased by confounding effects (e.g., patient motion or atrophy-induced PVEs). PVEs can actually be opposing, depending on the type of tracer used: for example, regional atrophy might lead to overestimation of cortical hypometabolism as measured by ${ }^{18} \mathrm{~F}-\mathrm{FDG}$ but to underestimation of amyloid tracer uptake as measured by ${ }^{11} \mathrm{C}-\mathrm{Pittsburgh}$ compound B. Simultaneous PET/MRI offers the unique option of carefully addressing these issues through accurate MRI-based motion and PVE correction. Furthermore, the exact relationship between PET and MRI findings can be investigated. This ability is of special interest because both

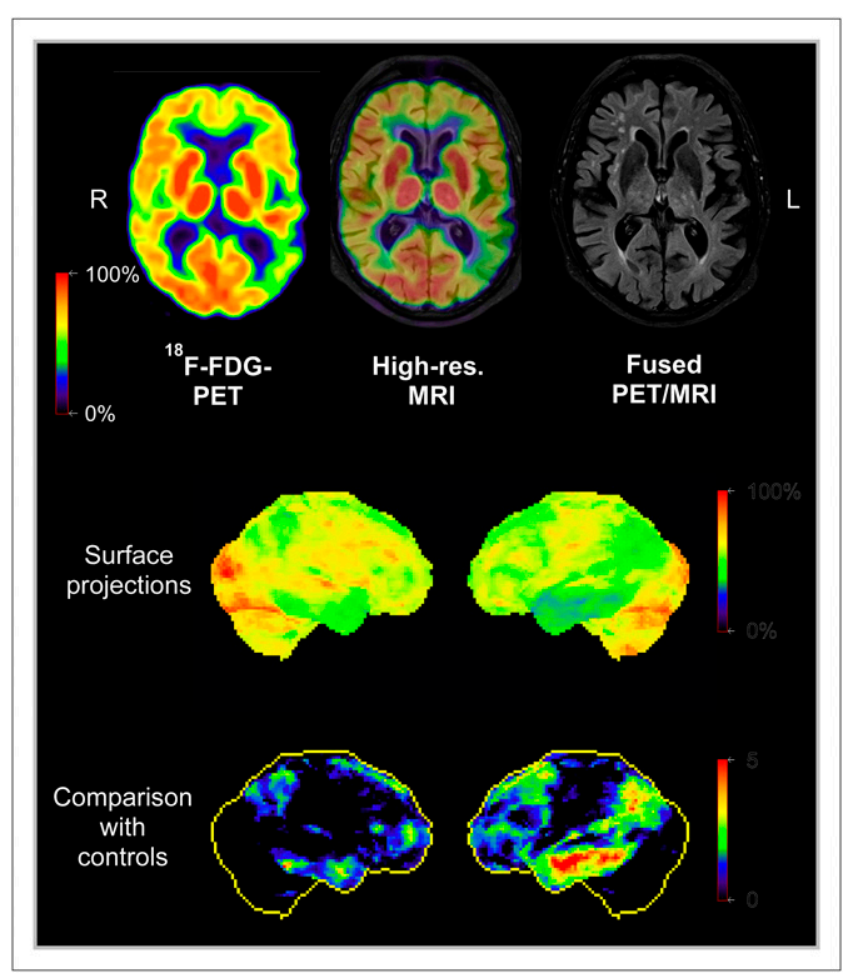

FIGURE 5. Simultaneous PET/MRI study in Alzheimer disease patient. (Top) Axial ${ }^{18} \mathrm{~F}-\mathrm{FDG}$ PET, high-resolution MRI, and fusion image. Areas with reduced metabolism (green) representing impaired neuronal function are visible in left temporoparietal cortex. (Middle and bottom) Surface projections of cerebral metabolism and of $z$ score images (comparison with controls). Data were acquired on Biograph mMR scanner at Nuklearmedizinische Klinik und Poliklinik der Technischen Universität München.

strong overlap (69) and considerable discrepancy between MRI structural changes and PET metabolic changes or amyloid deposits (70) have been demonstrated in previous studies on the development of Alzheimer disease $(71,72)$. Because atrophy is thought to represent a more downstream event in the course of neurodegeneration, the assessment of functional and molecular abnormalities together with the detection of the onset and extent of pathology may allow more precise staging of disease. In addition, simultaneous acquisition of resting-state fMRI, arterial spin labeling MRI, and PET may allow for a direct comparison between these functional parameters and for a cross-evaluation of their diagnostic value.

Deep brain stimulation (e.g., repetitive transcranial magnetic stimulation or direct current stimulation of selected cortical areas) has recently been suggested for the treatment of Alzheimer disease. Multiparametric functional imaging might improve our understanding of the effect of stimulation therapy on functional networks (fMRI) and neuroreceptor/transmitter systems (PET).

Finally, the opportunity to obtain complementary PET and MRI data simultaneously in a single imaging session is also likely to represent a major convenience, particularly in these often elderly and fragile patients. 


\section{Stroke/Cerebrovascular Disorders}

Based on the pioneering work performed with both PET and MRI in ischemic stroke, the concept of a potentially salvageable penumbra has emerged and is the most important (and today the only effective) basis for treatment of ischemic stroke. Therefore, an exact determination of potentially salvageable tissue is mandatory. Although PET methods are still considered a gold standard for noninvasively identifying the relevant regions of hemodynamic and metabolic compromise in ischemic stroke (73), PET is not routinely integrated into the firstline diagnostic evaluation of patients with suspected cerebrovascular issues or stroke, because the PET studies that would be the most useful in the acute phase of ischemic stroke (i.e., those involving ${ }^{15} \mathrm{O}$ - or ${ }^{11} \mathrm{C}$-labeled tracers) are also the most challenging logistically. In the acute setting, a fast diagnosis is needed because "time is brain," particularly regarding differentiation between ischemic and hemorrhagic strokes, which require very different therapeutic approaches. Diagnosis is usually obtained by CT or increasingly also by MRI $(74,75)$. The MRI perfusion/diffusion-weighted mismatch is believed to reflect tissue that is potentially salvageable (similar to the PET penumbra). However, perfusion-weighted imaging does not correctly estimate the PET penumbra; it demonstrates only hypoperfusion in the vascular bed. A direct comparison of simultaneously determined perfusion-weighted imaging hypoperfusion and low flow-preserved oxygensupplied tissue (increased oxygen extraction fraction) is necessary to really discriminate tissue that can benefit from recanalization therapies or to exclude patients who are not likely to benefit from thrombolytic therapy.

Simultaneous measurements might elucidate the ongoing debate concerning the relationship between the perfusiondiffusion mismatch and the PET penumbra (Fig. 6) (7679). PET/MRI cross-calibration and validation studies are likely to lead to the adoption of new perfusion-weighted imaging thresholds (80) that will allow a better selection of those patients who might still benefit from therapeutic interventions beyond the accepted 4.5 -h time window. As previously stated, "the time has come to calibrate simpler and widely applicable functional imaging procedures - especially diffusion- and perfusion-weighted MRI- on PET in order to make these modalities a reliable tool in the study of acute ischemic stroke" (76).

In the follow-up of ischemic stroke it is important to demonstrate the relationship between progression of a lesion (assessed using ${ }^{15} \mathrm{O}-\mathrm{PET}$ or microglial activation using translocator protein PET) and changes in perfusion- and diffusion-weighted images. Because these changes occur in small areas around the primary lesion and are transient, a simultaneous determination is necessary. In this context, activation studies (fMRI) would help to better understand the progression (or the recovery) of functional impairment in the surroundings of the ischemic lesion and within the functional network.

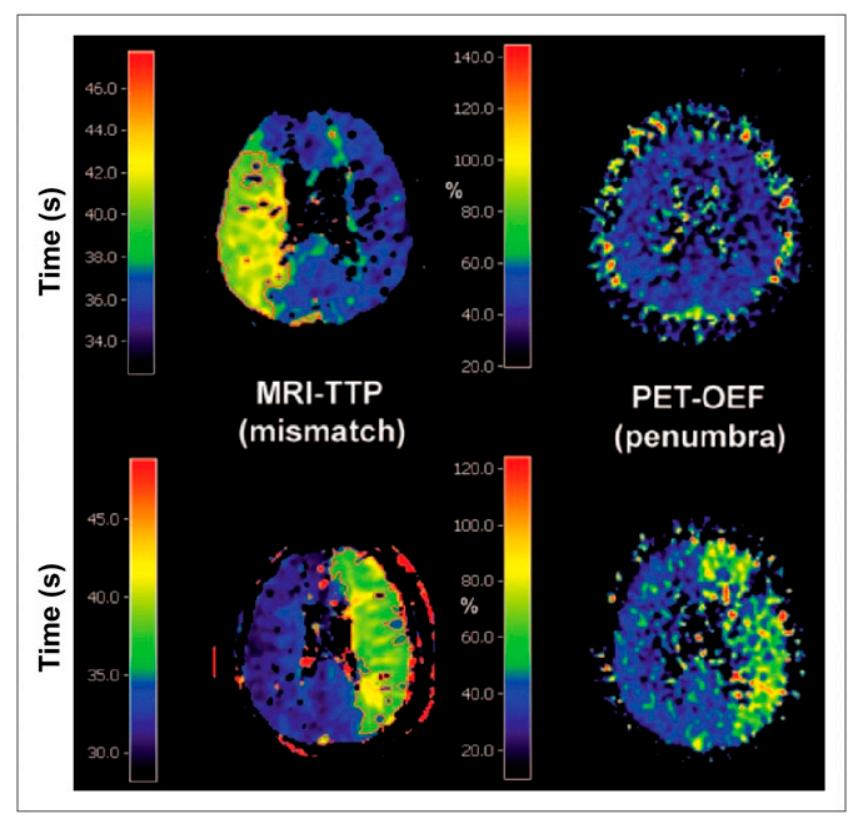

FIGURE 6. Comparison of MRI time-to-peak (TTP) and PET oxygen extraction fraction (OEF) images in 2 patients measured in chronic phase of stroke illustrating mismatch-penumbra debate. Disagreement between the 2 techniques is observed in first patient (top row), and agreement is observed in second patient (bottom row). (Reprinted with permission of (80).)

\section{Epilepsy}

Ictal-interictal SPECT is most often used for detecting the epileptogenic focus but relies on the presence of seizure activity. An imaging modality that allows the interictal detection of the lesion would be preferred, and combining the PET and MRI data (Fig. 7) might facilitate this task. It is known that there is a high probability that regional interictal hypometabolic lesions detected on ${ }^{18} \mathrm{~F}-$ FDG PET represent epileptogenic foci, even in MRInegative cases, or that the responsible focus can be selected from several structural abnormalities present on the MRI findings (81). In MRI-negative patients with temporal lobe epilepsy, ${ }^{11} \mathrm{C}$-flumazenil PET has been shown to be useful to detect the epileptic foci due to the reduced binding of this ligand to the inhibitory $\gamma$-aminobutyric acid receptors (82). Nevertheless, high-resolution MRI brain scans are required for focus localization, and precise coregistration of the PET and MRI volumes is important for planning surgery. The combination of PET and fMRI may also allow the detection of eloquent brain areas and topographic association with the epileptogenic material.

A major advantage of integrated PET/MRI for diagnosing epilepsy is that the number of times sedation or anesthesia is required might be reduced, particularly in younger children who cannot remain motionless during the scans. Furthermore, the reduced radiation when compared with PET/CT could be particularly useful for assessing medically intractable epilepsy in children. 


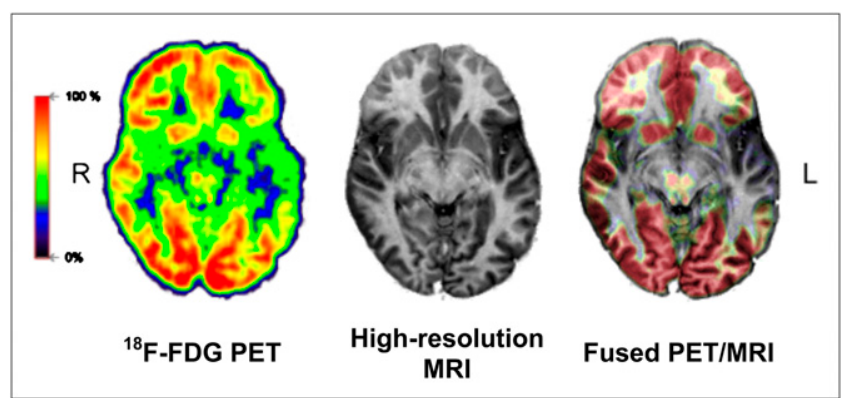

FIGURE 7. Simultaneous PET/MRI study in epilepsy patient. From left to right are shown axial FDG PET (60-75 min after injection), high-resolution MRI scan, and fusion image. Distinct hypometabolism is visible in polar region of left temporal lobe, typically corresponding to epileptogenic focus. Data were acquired on Biograph mMR scanner at Nuklearmedizinische Klinik und Poliklinik der Technischen Universität München.

\section{Neurobiology of Brain Activation}

Simultaneous PET/MRI may improve the understanding of the interaction between neurochemistry/transmitter-mediated processes and their functional, metabolic, and hemodynamic consequences in healthy brains and various disorders.

For example, in Parkinson disease, PET and MRI data might be used to simultaneously interrogate different phases of a complex pharmacologic response such as the rate of dopamine synthesis, release, transport, and receptor expression (PET) and neuronal activation (fMRI) after amphetamine stimulation (83).

Using an integrated scanner, the effect of deep brain stimulation of the nucleus accumbens (84) on metabolism and transmitter-receptor interaction in defined regions together with the connecting fiber tracts can be investigated. Similarly, one can study the effects of repetitive transcranial magnetic stimulation or of direct-current stimulation, which can be used to activate or inhibit selected areas of the cortex, on regional metabolism and on the involvement of transmitter-receptor systems with the connecting networks (85).

A highly interesting scientific option is the simultaneous measurement of neurochemical changes (PET) and changes in regional brain activity (BOLD-fMRI), including the effects of activation by specific tasks on transmitter release or receptor binding. For example, during learning of motor skills, the effects of regional neuronal activation might be dynamically monitored by BOLD-fMRI and, simultaneously, might be detected by a PET study in which a dopamine receptor ligand is displaced from the receptor site (86). By analogy, the response to pain with regard to neuronal activation and, for example, opiate receptor displacement might be assessed simultaneously. Findings from such simultaneous multimodal imaging might also be related to task performance, behavior, hormone levels, and subjective perception, among others. Because neurons represent the fastest-acting cells in the human body, sequential imaging approaches may never be able to truly reproduce a situation and to synchronize the findings from the 2 modalities. Perfusion, metabolism, neuronal activity, receptor/ transmitter status, and functional connectivity may change within seconds. Furthermore, cerebral processes are affected by mood, hormonal status, vigilance, or levels of medication, which can change within minutes. Finally, habituation may lead to differences in the performance of repeated tasks (or their cerebral processing), as well as to changes in expectation or in the subjective evaluation of any repeated sensation. Thus, in a sequential approach, it neither will be possible to establish a temporal relation between PET and MRI findings nor can it be ensured that the brain processes investigated are comparable. In contrast, simultaneous PET/MRI may open totally new perspectives in the analysis of neurochemical and electrophysiologic interactions in the brain.

\section{Translational Research}

Tremendous progress is constantly being made in the molecular and cellular imaging field. Integrated PET/MRI will be of great value for the transfer of these findings to humans and the development of innovative treatment strategies. Examples of applications that might benefit from simultaneous assessment of various parameters are monitoring the therapeutic effects of targeted gene transfer, stem cell transplantation, and cell replacement approaches.

Targeted genes can be transferred using various vectors for induction of foreign enzyme expression in selected cells. These foreign enzymes can render the induced cells susceptible to specific drugs, which then are converted into toxic compounds. This approach was applied for experimental treatment of glioblastoma by transferring the herpes simplex thymidine kinase gene, which made the cells susceptible to ganciclovir. The expression of the transferred gene and the effect on tumor metabolism and growth could be followed by PET and MRI (87).

Simultaneous PET/MRI can be used to show the viability and differentiation of transplanted cells and their effect on the neuronal network. Embryonic stem cells can be implanted into brain lesions; for example, the destroyed striatum and the proliferation and differentiation of dopaminergic cells can be followed using combined measurements. The restored functional activity of the transplanted cells can be demonstrated by the response to amphetamine, which causes an increase in relative CBV due to dopamine release (88).

Cell replacement approaches have been proposed for treatment of various neurologic disorders (e.g., ischemic stroke). For this strategy, it is essential to monitor the location and follow the migration of grafted stem or progenitor cells. Various methods can be used for labeling these cells, making them detectable by MRI (e.g., iron oxide particles) (89). The migration of these cells to the border zone of damaged brain tissue in the affected hemisphere can be followed by MRI. Monitoring viability and migration, as well as integration of cells into functional networks, by simultaneous PET/MRI might become a qualifying step in strategies relying on transplantation of fetal grafts in various neurologic diseases. 


\section{CONCLUSION}

For years, simultaneous PET/MRI was thought of as a potential Holy Grail of molecular imaging, and of molecular neuroimaging in particular. Integrated PET/MRI offers several promising options regarding the clinical evaluation of disorders of the brain. The high diagnostic value of MRI and PET procedures for neuropsychiatric conditions may turn PET/MRI into the hybrid imaging modality of choice for diagnostic questions directed toward pathologies inside the brain. PET/MRI may have an improved diagnostic value, compared with the value of each of the individual procedures, and may optimize workflow and increase patient comfort.

From a scientific perspective, PET/MRI should improve our understanding of healthy-brain physiology and function and open new insights into the pathophysiologic interrelation between different parameters involved in disorders of the central nervous system. The clever design and analysis of data obtained with specific tracers will allow for estimation of changes in receptor binding in response to a stimulus, a concept directly complementary to fMRI activation studies to investigate brain physiology. As we probe illnesses of the mind more thoroughly, the state of the mind and the state of the brain at any given instant become more relevant. In our quest for understanding how our mind works, the great opportunity (and challenge) created by simultaneous PET/MRI is to use dynamic measures of brain physiology from MRI and new generations of receptor-specific tracers (with long plasma half-lives and rapidly reversible displaceability) to perform receptor-specific activation mapping using fMRI-like paradigms.

Although a range of existing studies requiring data from both modalities might benefit from this new imaging technique, we expect that the most exciting opportunities will emerge from new molecular neuroimaging applications that will provide scientific and medical benefits.

\section{DISCLOSURE}

Dr. Catana has received honoraria from Siemens Healthcare for lectures. The authors of this article have indicated no other relevant relationships that could be perceived as a real or apparent conflict of interest.

\section{REFERENCES}

1. Schlemmer H-PW, Pichler BJ, Schmand M, et al. Simultaneous MR/PET imaging of the human brain: feasibility study. Radiology. 2008;248:1028-1035.

2. Drzezga A, Souvatzoglou M, Eiber M, et al. First clinical experience with integrated whole-body PET/MR: comparison to PET/CT in patients with oncologic diagnoses. J Nucl Med. 2012;53:845-855.

3. Picard Y, Thompson CJ. Motion correction of PET images using multiple acquisition frames. IEEE Trans Med Imaging. 1997;16:137-144.

4. Fulton RR, Meikle SR, Eberl S, Pfeiffer J, Constable CJ, Fulham MJ. Correction for head movements in positron emission tomography using an optical motiontracking system. IEEE Trans Nucl Sci. 2002;49:116-123.

5. Bloomfield PM, Spinks TJ, Reed J, et al. The design and implementation of a motion correction scheme for neurological PET. Phys Med Biol. 2003;48:959-978.

6. Carson RE, Barker WC, Liow J-S, Johnson CA. Design of a motion-compensation OSEM list-mode algorithm for resolution-recovery reconstruction for the HRRT. Nuclear Science Symposium Conference Record, IEEE. 2003;5:3281-3285.
7. Bühler P, Just U, Will E, Kotzerke J, van den Hoff J. An accurate method for correction of head movement in PET. IEEE Trans Med Imaging. 2004;23:1176-1185.

8. Rahmim A, Bloomfield P, Houle S, et al. Motion compensation in histogrammode and list-mode EM reconstructions: beyond the event-driven approach. IEEE Trans Nucl Sci. 2004;51:2588-2596.

9. Herzog H, Tellmann L, Fulton R, et al. Motion artifact reduction on parametric PET images of neuroreceptor binding. J Nucl Med. 2005;46:1059-1065.

10. Raghunath N, Faber TL, Suryanarayanan S, Votaw JR. Motion correction of PET brain images through deconvolution: II. Practical implementation and algorithm optimization. Phys Med Biol. 2009;54:813-829.

11. Olesen OV, Paulsen RR, Højgaard L, Roed B, Larsen R. Motion tracking for medical imaging: a nonvisible structured light tracking approach. IEEE Trans Med Imaging. 2012;31:79-87.

12. van der Kouwe AJ, Benner T, Dale AM. Real-time rigid body motion correction and shimming using cloverleaf navigators. Magn Reson Med. 2006;56:1019-1032.

13. Catana C, Benner T, van der Kouwe A, et al. MRI-assisted PET motion correction for neurologic studies in an integrated MR-PET scanner. J Nucl Med. 2011;52:154-161.

14. Catana C, van der Kouwe A, Benner T, et al. Toward implementing an MRIbased PET attenuation-correction method for neurologic studies on the MR-PET brain prototype. J Nucl Med. 2010;51:1431-1438.

15. Hoffman EJ, Huang SC, Phelps ME. Quantitation in positron emission computedtomography. 1. Effect of object size. J Comput Assist Tomogr. 1979;3:299-308.

16. Meltzer CC, Leal JP, Mayberg HS, Wagner HN, Frost JJ. Correction of PET data for partial volume effects in human cerebral cortex by MR imaging. J Comput Assist Tomogr. 1990;14:561-570.

17. Müller-Gärtner HW, Links JM, Prince JL, et al. Measurement of radiotracer concentration in brain gray-matter using positron emission tomography: MRI-based correction for partial volume effects. J Cereb Blood Flow Metab. 1992;12:571-583.

18. Labbé C, Koepp M, Ashburner J, et al. Absolute PET quantification with correction for partial volume effects within cerebral structures. In: Carson R, DaubeWitherspoon M, Herscovitch P, eds. Quantitative Functional Brain Imaging with Positron Emission Tomography. San Diego, CA: Academic Press; 1998:59-66.

19. Rousset OG, Ma YL, Evans AC. Correction for partial volume effects in PET: principle and validation. J Nucl Med. 1998;39:904-911.

20. Kirov AS, Piao JZ, Schmidtlein CR. Partial volume effect correction in PET using regularized iterative deconvolution with variance control based on local topology. Phys Med Biol. 2008;53:2577-2591.

21. Leahy R, Yan X. Incorporation of anatomical MR data for improved functional imaging with PET. In: Hawkes D, Colchester A, eds. Information Processing in Medical Imaging. New-York, NY: Wiley-Liss; 1991:105-120.

22. Wang $\mathrm{CH}$, Chen JC, Kao CM, Liu RS. Incorporation of correlated MR images in MAP reconstruction of PET images [abstract]. J Nucl Med. 2003;44(suppl):278P.

23. Baete K, Nuyts J, Van Laere K, et al. Evaluation of anatomy based reconstruction for partial volume correction in brain FDG-PET. Neuroimage. 2004;23:305-317.

24. Rousset OG, Ma YL, Wong DF, Evans AC. Pixel- versus region-based partial volume correction in PET. In: Carson R, Daube-Witherspoon M, Herscovitch P, eds. Quantitative Functional Brain Imaging with Positron Emission Tomography. San Diego, CA: Academic Press; 1998:67-75.

25. Strul D, Bendriem B. Robustness of anatomically guided pixel-by-pixel algorithms for partial volume effect correction in positron emission tomography. J Cereb Blood Flow Metab. 1999;19:547-559.

26. Zanotti-Fregonara P, Chen K, Liow JS, Fujita M, Innis RB. Image-derived input function for brain PET studies: many challenges and few opportunities. J Cereb Blood Flow Metab. 2011;31:1986-1998.

27. Villringer A, Rosen BR, Belliveau JW, et al. Dynamic imaging with lanthanide chelates in normal brain: contrast due to magnetic-susceptibility effects. Magn Reson Med. 1988;6:164-174.

28. Williams DS, Detre JA, Leigh JS, Koretsky AP. Magnetic-resonance-imaging of perfusion using spin inversion of arterial water. Proc Natl Acad Sci USA. 1992;89:212-216.

29. Donahue MJ, Lu H, Jones C, Pekar J, van Zijl P. An account of the discrepancy between MRI and PET cerebral blood flow measures: a high-field MRI investigation. NMR Biomed. 2006;19:1043-1054.

30. Kwong KK, Belliveau JW, Chesler DA, et al. Dynamic MRI of human brain activity during primary sensory stimulation. Proc Natl Acad Sci USA. 1992;89:5675-5679.

31. Belliveau JW, Kennedy DN, McKinstry RC, et al. Functional mapping of the human visual-cortex by magnetic-resonance-imaging. Science. 1991;254:716-719.

32. Fox PT, Raichle ME. Focal physiological uncoupling of cerebral blood-flow and oxidative-metabolism during somatosensory stimulation in human subjects. Proc Natl Acad Sci USA. 1986;83:1140-1144.

33. Ogawa S, Lee TM, Kay AR, Tank DW. Brain magnetic-resonance-imaging with contrast dependent on blood oxygenation. Proc Natl Acad Sci USA. 1990;87: 9868-9872. 
34. Bolar DS. Magnetic Resonance Imaging of the Cerebral Metabolic Rate of Oxygen $\left(\mathrm{CMRO}_{2}\right)$. Cambridge, MA: Harvard University-MIT Division of Health Sciences and Technology, Massachusetts Institute of Technology; 2010.

35. Fan AP, Benner T, Bolar DS, Rosen BR, Adalsteinsson E. Phase-based regional oxygen metabolism (PROM) using MRI. Magn Reson Med. 2012;67:669-678.

36. Biswal B, Yetkin FZ, Haughton VM, Hyde JS. Functional connectivity in the motor cortex of resting human brain using echo-planar MRI. Magn Reson Med. 1995;34:537-541.

37. Drzezga A, Becker JA, Van Dijk KRA, et al. Neuronal dysfunction and disconnection of cortical hubs in non-demented subjects with elevated amyloid burden. Brain. 2011;134:1635-1646.

38. Sorensen AG. Magnetic resonance as a cancer imaging biomarker. J Clin Oncol. 2006;24:3274-3281.

39. Hylton N. Dynamic contrast-enhanced magnetic resonance imaging as an imaging biomarker. J Clin Oncol. 2006;24:3293-3298.

40. Hamstra DA, Rehemtulla A, Ross BD. Diffusion magnetic resonance imaging: a biomarker for treatment response in oncology. J Clin Oncol. 2007;25:4104-4109.

41. Aronen HJ, Gazit IE, Louis DN, et al. Cerebral blood-volume maps of gliomas: comparison with tumor grade and histologic findings. Radiology. 1994;191:41-51.

42. Tofts PS, Brix G, Buckley DL, et al. Estimating kinetic parameters from dynamic contrast-enhanced T(1)-weighted MRI of a diffusable tracer: standardized quantities and symbols. J Magn Reson Imaging. 1999;10:223-232.

43. Jain RK, Duda DG, Clark JW, Loeffler JS. Lessons from phase III clinical trials on anti-VEGF therapy for cancer. Nat Clin Pract Oncol. 2006;3:24-40.

44. Muzi M, Spence AM, O'Sullivan F, et al. Kinetic analysis of 3'-deoxy-3'-F-18fluorothymidine in patients with gliomas. J Nucl Med. 2006;47:1612-1621.

45. Schiepers C, Dahlbom M, Chen W, et al. Kinetics of 3'-deoxy-3'-F-18-fluorothymidine during treatment monitoring of recurrent high-grade glioma. $\mathrm{J} \mathrm{Nucl}$ Med. 2010;51:720-727.

46. Thorwarth D, Eschmann SM, Paulsen F, Alber M. A kinetic model for dynamic [F-18]Fmiso PET data to analyse tumour hypoxia. Phys Med Biol. 2005;50:2209-2224.

47. Vredenburgh JJ, Desjardins A, Herndon JE II, et al. Bevacizumab plus irinotecan in recurrent glioblastoma multiforme. J Clin Oncol. 2007;25:4722-4729.

48. Sathornsumetee S, Cao Y, Marcello JE, et al. Tumor angiogenic and hypoxic profiles predict radiographic response and survival in malignant astrocytoma patients treated with bevacizumab and irinotecan. J Clin Oncol. 2008;26:271-278.

49. Heiss WD, Raab P, Lanfermann H. Multimodality assessment of brain tumors and tumor recurrence. $J$ Nucl Med. 2011;52:1585-1600.

50. Grosu AL, Weber WA, Franz M, et al. Reirradiation of recurrent high-grade gliomas using amino acid PET (SPECT)/CT/MRI image fusion to determine gross tumor volume for stereotactic fractionated radiotherapy. Int J Radiat Oncol Biol Phys. 2005;63:511-519.

51. Thiel A, Habedank B, Herholz K, et al. From the left to the right: how the brain compensates progressive loss of language function. Brain Lang. 2006;98:57-65.

52. Ashburner J, Friston KJ. Voxel-based morphometry: the methods. Neuroimage. 2000;11:805-821.

53. Karas GB, Burton EJ, Rombouts S, et al. A comprehensive study of gray matter loss in patients with Alzheimer's disease using optimized voxel-based morphometry. Neuroimage. 2003;18:895-907.

54. Chételat G, Landeau B, Eustache F, et al. Using voxel-based morphometry to map the structural changes associated with rapid conversion in MCI: a longitudinal MRI study. Neuroimage. 2005;27:934-946.

55. Fischl B, Dale AM. Measuring the thickness of the human cerebral cortex from magnetic resonance images. Proc Natl Acad Sci USA. 2000;97:11050-11055.

56. Du AT, Schuff N, Kramer JH, et al. Different regional patterns of cortical thinning in Alzheimer's disease and frontotemporal dementia. Brain. 2007;130:1159-1166.

57. Bozzali M, Falini A, Franceschi M, et al. White matter damage in Alzheimer's disease assessed in vivo using diffusion tensor magnetic resonance imaging. J Neurol Neurosurg Psychiatry. 2002;72:742-746.

58. Chua TC, Wen W, Slavin MJ, Sachdev PS. Diffusion tensor imaging in mild cognitive impairment and Alzheimer's disease: a review. Curr Opin Neurol. 2008;21:83-92.

59. Dickerson BC, Sperling RA. Functional abnormalities of the medial temporal lobe memory system in mild cognitive impairment and Alzheimer's disease: insights from functional MRI studies. Neuropsychologia. 2008;46:1624-1635.

60. Sperling RA, Dickerson BC, Pihlajamaki M, et al. Functional alterations in memory networks in early Alzheimer's disease. Neuromolecular Med. 2010;12:27-43.

61. Buckner RL, Andrews-Hanna JR, Schacter DL. The brain's default network: anatomy, function, and relevance to disease. Year in Cognitive Neuroscience 2008. Vol 1124. Oxford, U.K.: Blackwell Publishing; 2008:1-38.

62. Greicius MD, Srivastava G, Reiss AL, Menon V. Default-mode network activity distinguishes Alzheimer's disease from healthy aging: evidence from functional MRI. Proc Natl Acad Sci USA. 2004;101:4637-4642.
63. Bohnen NI, Djang DSW, Herholz K, Anzai Y, Minoshima S. Effectiveness and safety of ${ }^{18}$ F-FDG PET in the evaluation of dementia: a review of the recent literature. J Nucl Med. 2012;53:59-71.

64. Pappatà $\mathrm{S}$, Salvatore $\mathrm{E}$, Postiglione $\mathrm{A}$. In vivo imaging of neurotransmission and brain receptors in dementia. J Neuroimaging. 2008;18:111-124.

65. Kendziorra K, Wolf H, Meyer PM, et al. Decreased cerebral alpha 4 beta $2 *$ nicotinic acetylcholine receptor availability in patients with mild cognitive impairment and Alzheimer's disease assessed with positron emission tomography. Eur J Nucl Med Mol Imaging. 2011;38:515-525.

66. Herholz K, Weisenbach S, Zundorf $\mathrm{G}$, et al. In vivo study of acetylcholine esterase in basal forebrain, amygdala, and cortex in mild to moderate Alzheimer disease. Neuroimage. 2004;21:136-143.

67. Klunk WE, Engler H, Nordberg A, et al. Imaging brain amyloid in Alzheimer's disease with Pittsburgh compound-B. Ann Neurol. 2004;55:306-319.

68. Rowe CC, Villemagne VL. Brain amyloid imaging. J Nucl Med. 2011;52:1733-1740.

69. Buckner RL, Snyder AZ, Shannon BJ, et al. Molecular, structural, and functional characterization of Alzheimer's disease: evidence for a relationship between default activity, amyloid, and memory. J Neurosci. 2005;25:7709-7717.

70. Drzezga A, Grimmer T, Henriksen G, et al. Effect of APOE genotype on amyloid plaque load and gray matter volume in Alzheimer disease. Neurology. 2009;72:1487-1494.

71. Drzezga A, Lautenschlager N, Siebner H, et al. Cerebral metabolic changes accompanying conversion of mild cognitive impairment into Alzheimer's disease: a PET follow-up study. Eur J Nucl Med Mol Imaging. 2003;30:1104-1113.

72. Mosconi L, Tsui WH, De Santi S, et al. Reduced hippocampal metabolism in MCI and AD: automated FDG-PET image analysis. Neurology. 2005;64:1860-1867.

73. Heiss WD. Ischemic penumbra: evidence from functional imaging in man. J Cereb Blood Flow Metab. 2000;20:1276-1293.

74. Lövblad KO, Laubach HJ, Baird AE, et al. Clinical experience with diffusionweighted MR in patients with acute stroke. Am J Neuroradiol. 1998;19:1061-1066.

75. Sorensen AG, Copen WA, Ostergaard L, et al. Hyperacute stroke: simultaneous measurement of relative cerebral blood volume, relative cerebral blood flow, and mean tissue transit time. Radiology. 1999;210:519-527.

76. Heiss WD. Best measure of ischemic penumbra: positron emission tomography. Stroke. 2003;34:2534-2535.

77. Warach S. Measurement of the ischemic penumbra with MRI: It's about time. Stroke. 2003;34:2533-2534.

78. Guadagno JV, Warburton EA, Jones PS, et al. How affected is oxygen metabolism in DWI lesions? A combined acute stroke PET-MR study. Neurology. 2006;67:824-829.

79. Takasawa M, Jones PS, Guadagno JV, et al. How reliable is perfusion MR in acute stroke? Validation and determination of the penumbra threshold against quantitative PET. Stroke. 2008;39:870-877.

80. Sobesky J, Weber OZ, Lehnhardt FG, et al. Which time-to-peak threshold best identifies penumbral flow? A comparison of perfusion-weighted magnetic resonance imaging and positron emission tomography in acute ischemic stroke. Stroke. 2004;35:2843-2847.

81. LoPinto-Khoury C, Sperling MR, Skidmore C, et al. Surgical outcome in PETpositive, MRI-negative patients with temporal lobe epilepsy. Epilepsia. 2012;53:342-348.

82. Hammers A, Koepp MJ, Hurlemann R, et al. Abnormalities of grey and white matter $\left[{ }^{11} \mathrm{C}\right]$ flumazenil binding in temporal lobe epilepsy with normal MRI. Brain. 2002;125:2257-2271.

83. Chen YCI, Galpern WR, Brownell AL, et al. Detection of dopaminergic neurotransmitter activity using pharmacologic MRI: correlation with PET, microdialysis, and behavioral data. Magn Reson Med. 1997;38:389-398.

84. van Kuyck K, Gabriels L, Cosyns P, et al. Behavioural and physiological effects of electrical stimulation in the nucleus accumbens: a review. Acta Neurochir Suppl. 2007;97:375-391.

85. Thiel A, Schumacher B, Wienhard K, et al. Direct demonstration of transcallosal disinhibition in language networks. J Cereb Blood Flow Metab. 2006;26:1122-1127.

86. Badgaiyan RD, Fischman AJ, Alpert NM. Striatal dopamine release in sequential learning. Neuroimage. 2007;38:549-556.

87. Jacobs A, Voges J, Reszka R, et al. Positron-emission tomography of vector-mediated gene expression in gene therapy for gliomas. Lancet. 2001;358:727-729.

88. Bjorklund LM, Sanchez-Pernaute R, Chung SM, et al. Embryonic stem cells develop into functional dopaminergic neurons after transplantation in a Parkinson rat model. Proc Natl Acad Sci USA. 2002;99:2344-2349.

89. Hoehn M, Wiedermann D, Justicia C, et al. Cell tracking using magnetic resonance imaging. J Physiol. 2007;584:25-30. 